Davide Anderegg

\title{
Digitale Anhörungen für den Kanton Aargau
}

Per Ende September 2020 hat der Kanton Aargau den Prozess zu öffentlichen kantonalen Anhörungen und Vernehmlassungen vollständig digitalisiert. Was steckt hinter dem Projekt mit dem Namen «eAnhörungen» und wer zieht welchen Nutzen daraus? Der Werkstattbericht gibt Einblick in das digitale Pionierprojekt des Kantons Aargau.

Beitragsart: Werkstattberichte

Zitiervorschlag: Davide Anderegg, Digitale Anhörungen für den Kanton Aargau, in: LeGes 31 (2020) 3 


\section{Inhaltsübersicht}

1. Fokus auf den Anhörungsfragebogen

1.1. Hürden für die Teilnehmerinnen und Teilnehmer an einer Anhörung ...

1.2. ....und für die Stelle, die eine Anhörung durchführt

2. Der Kanton Aargau auf dem digitalen Weg

3. Intensiver Erarbeitungsprozess von Anbeginn des Projekts

3.1. Oberstes Gebot: Keine Informationen verstecken

3.2. Neue Möglichkeiten für die Teilnehmerinnen und Teilnehmer an einer Anhörung ...

3.3. ... und für die kantonale Verwaltung

4. Erste Erfahrungen

5. Einschränkungen

6. Bilanz und Ausblick

[1] Die Anhörung, andernorts auch «Vernehmlassungsverfahren» genannt, ist eine wichtige und zwingende Etappe auf dem Weg zur Entstehung eines Gesetzes oder eines anderen referendumspflichten Beschlusses ( $§ 66$ Abs. 2 KV-AG). Ziel der Anhörung ist, dass sachlich taugliche und politisch tragbare Entwürfe zustande kommen. Wie alle Kantone führt auch der Kanton Aargau regelmässig Anhörungen durch. Damit wird die Mitwirkung von Parteien, Interessensgruppen und der Öffentlichkeit bei Erlassen sichergestellt.

\section{Fokus auf den Anhörungsfragebogen}

[2] Eines der wichtigsten Instrumente bei Anhörungen des Kantons Aargau stellt der Fragebogen dar. Anhand der darin notierten Fragen werden die verschiedenen Stellungnahmen inhaltlich strukturiert abgeholt; zudem können die Anhörungsteilnehmenden im Fragebogen ihre Bemerkungen und Anregungen festhalten.

\subsection{Hürden für die Teilnehmerinnen und Teilnehmer an einer Anhörung ...}

[3] In Zeiten der Digitalisierung bot dieser Fragebogen für die Anhörungsteilnehmenden nur einen geringen Mehrwert. Der Fragebogen wurde zwar als ausfüllbares PDF-Dokument angeboten, es ergaben sich teilweise jedoch Schwierigkeiten beim Öffnen des Dokuments. Auch das Einreichen einer Stellungnahme im Namen einer Gruppierung oder Organisation war nicht sonderlich attraktiv, das Dokument musste zur Erarbeitung der Stellungnahme häufig hin und her geschickt werden, was Mehrfachablagen bei den Betroffenen begünstigte. Ebenso wurden nicht alle Stellungnahmen digital, sondern teilweise per Post oder als Scan eingereicht, was verwaltungsintern ein aufwendiges digitales Überführen nötig machte.

\section{2. ... und für die Stelle, die eine Anhörung durchführt}

[4] Der Anhörungsfragebogen bot auch Stolpersteine für die kantonale Verwaltung. Für das Erstellen des ausfüllbaren PDFs war technisches Knowhow nötig. Die eingereichten Stellungnah- 
men standen ausserdem nur als Daten in Excel zur Verfügung, was die Auswertung zeitintensiv machte, wenn man sie für die verschiedenen Berichte und Botschaften weiterverwenden wollte.

[5] Grund genug, hier Abhilfe zu schaffen!

\section{Der Kanton Aargau auf dem digitalen Weg}

[6] Die Idee, den Anhörungsprozess zu digitalisieren, wurde im Sommer 2018 konkretisiert und fiel in der kantonalen Verwaltung auf fruchtbaren Boden. Denn die Aargauer Regierung hatte bereits seit geraumer Zeit beschlossen, die Digitalisierung aktiv zu nutzen, um Mehrwerte für die Bevölkerung und die Wirtschaft zu generieren. Konkret beschleunigt der Regierungsrat mit der Strategie «Digitale Transformation SmartAargau» seit mehreren Jahren die Modernisierung der Verwaltung und fördert gezielt Vereinfachungen und Digitalisierungsvorhaben. Der Auftrag des Projekts unter dem Titel «eAnhörungen» lautete schliesslich, dass öffentliche Anhörungen des Kantons Aargau künftig digital über das Online-Bürgerkonto «Mein Konto» durchgeführt werden sollen.

\begin{tabular}{l}
\hline "Mein Konto" \\
"Mein Konto" ist die offizielle \\
E-Government-Transaktionsplattform \\
des Kantons Aargau. Wer sich regi- \\
striert, kann rund um die Uhr per \\
Internet spezifische Verwaltungs- \\
dienstleistungen zu nutzen. \\
\end{tabular}

\section{Intensiver Erarbeitungsprozess von Anbeginn des Projekts}

[7] Die gesamte Erarbeitungsphase von eAnhörungen dauerte rund zwei Jahre. In das Projekt wurden wichtige Adressaten von Anhörungen einbezogen. Hierfür wurde initial eine Umfrage bei über 300 Organisationen (Parteien, Verbände, Gemeinden etc.) durchgeführt, um mehr darüber zu erfahren, wie Anhörungsantworten ausgearbeitet werden und welche Wünsche und Bedürfnisse vorhanden sind. Die Leitung des Projekts eAnhörungen übernahm das Departement Bildung, Kultur und Sport; alle weiteren Departemente und die Staatskanzlei des Kantons Aargau waren eng in das Projekt eingebunden. Der externe Umfrageanbieter Survalyzer war ebenfalls von Anfang an mit an Bord. 


\subsection{Oberstes Gebot: Keine Informationen verstecken}

[8] Die Umfrage legte die Basis dazu, wichtige Adressaten laufend über das Projekt zu informieren. Eine Projekt-Website trug ebenfalls dazu bei, dass stets Transparenz betreffend möglicher Neuerungen im Rahmen des Projekts eAnhörungen herrschte: Hier wurde das Projekt vorgestellt, ein Erklärvideo aufgeschaltet und oft genannte Fragen und Antworten wurden gebündelt. Im Hinblick auf die Umsetzung und die Akzeptanz des Projekts war dies zentral, schliesslich musste der neue Anhörungsprozess von den Betroffenen angenommen und verstanden werden.

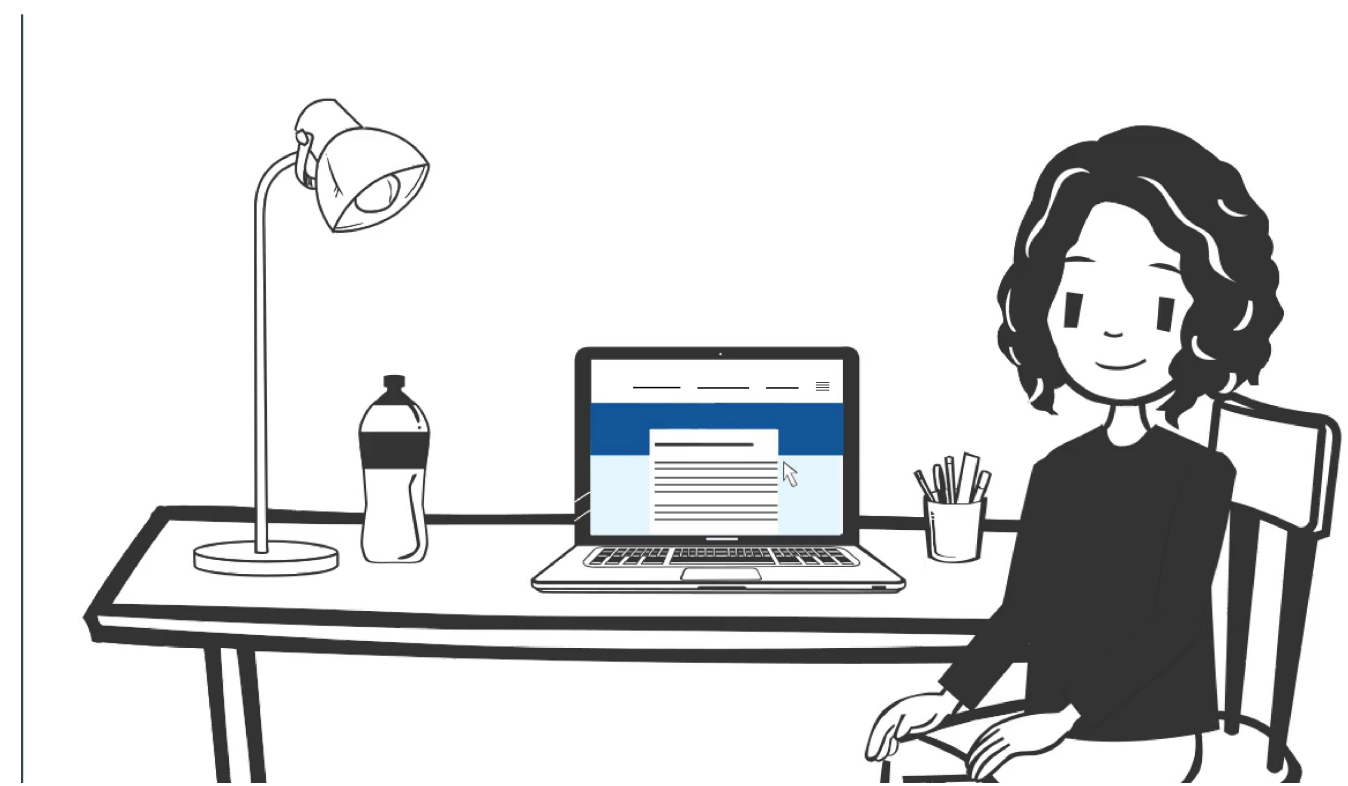

[Standbild Erklärvideo:] Mit einem Erklärvideo wurde das Projekt eAnhörungen gegenüber wichtigen Adressaten und allen Interessierten bildhaft vorgestellt. Foto: $z \mathrm{Vg}$.

[9] Von Anfang an war eines der obersten Gebote des Projekts, dass sich an der Zugänglichkeit der Informationen zu öffentlichen Anhörungen nichts ändert. Weiterhin sollten alle Informationen zu einer Anhörung - wie die Adressatenliste, der Anhörungsbericht, Beilagen, sogar die Fragen zur Anhörung - frei und nach heutigem Prinzip einsehbar sein. Weiterhin finden alle Interessierten unter www.ag.ch/anhörungen alle Informationen zu laufenden eAnhörungen. Erst wenn man den Fragebogen einer Anhörung beantworten möchte, folgt eine Aufforderung zum Login ins Bürgerkonto.

\subsection{Neue Möglichkeiten für die Teilnehmerinnen und Teilnehmer an einer Anhörung ...}

[10] Mit dem Projekt eAnhörungen wurden im Kanton Aargau mehrere Neuerungen geschaffen. Zentrales Element von eAnhörungen stellt der Anhörungsfragebogen dar, der nicht mehr als elektronisches PDF-Dokument, sondern als Online-Fragebogen mit Survalyzer umgesetzt wird, der in «Mein Konto» integriert ist. Dadurch ergeben sich für die Anhörungsteilnehmenden mehrere Vorzüge mit eAnhörungen, so zum Beispiel: 
- Vollständig digital eine Anhörung beantworten

- Online-Zwischenspeichern angefangener Stellungnahmen

- Einfaches Wechseln zwischen der Beantwortung einer Anhörung als Privatperson oder im Namen einer Organisation

- Gemeinsames Ausarbeiten von Stellungnahmen im Namen einer Organisation durch mehrere Personen

- Online-Zugriff auf bereits eingereichte eigene Stellungnahmen

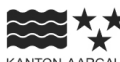

Davide Anderegg () Suche Q Menü

\section{eAnhörungen}

i Informationen zur Tabellen-Ansicht

Sie sehen unten laufende eAnhörungen und Ihre eingereichten Stellungnahmen. Bis Ende 2020 ist es möglich, dass noch nicht alle öffentlichen Anhörungen als eAnhörungen durchgeführt werden. Die Gesamtübersicht finden Sie unter den laufenden Anhörungen.

$\rightarrow$ Zu allen laufenden Anhörungen

Liste nach Status filtern

$$
\begin{aligned}
& \square \text { Eingeladen } \\
& \square \text { Ohne Einladung } \\
& \square \text { In Bearbeitung } \\
& \square \text { Eingereicht }
\end{aligned}
$$

\begin{tabular}{|c|c|c|c|c|c|}
\hline EANHÖRUNGEN & BEGINN & ENDE & ORGANISATION & STATUS & $\begin{array}{l}\text { FRAGEBOGEN } \\
\text { HERUNTERLADEN }\end{array}$ \\
\hline $\begin{array}{l}\text { Steuergesetz (StG); Änderung } \\
\text { Regierungsrat eröffnet Zusatz-Anhörung für Steuergesetz- } \\
\text { Revision } 2022\end{array}$ & 30.10 .2020 & 08.01.2021 & DFR & $\begin{array}{l}\text { Ohne Einladung } \\
\text { Teilnehmen }\end{array}$ & - \\
\hline $\begin{array}{l}\text { Neubau Amt für Verbraucherschutz; Verpflichtungskredit } \\
\text { Bauprojekt } \\
\text { Für den Neubau des Amts für Verbraucherschutz soll dem } \\
\text { Grossen Rat ein Baukredit von total } 48,46 \text { Millionen Franken } \\
\text { beantragt werden. }\end{array}$ & 23.10.2020 & 20.01.2021 & DGS & $\begin{array}{l}\text { In Bearbeitung } \\
\text { Fortfahren } \\
\text { Löschen }\end{array}$ & 串 \\
\hline
\end{tabular}

[Übersicht eAnhörungen:] In der Übersicht zu eAnhörungen können Anhörungsteilnehmende unter anderem direkt in den Fragebogen einsteigen oder erkennen, ob sie die Beantwortung bereits begonnen und unterbrochen haben. Foto: $z \mathrm{Vg}$. 


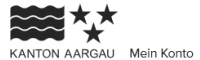

Davide Anderegg @) Suche Q Menü $\equiv$

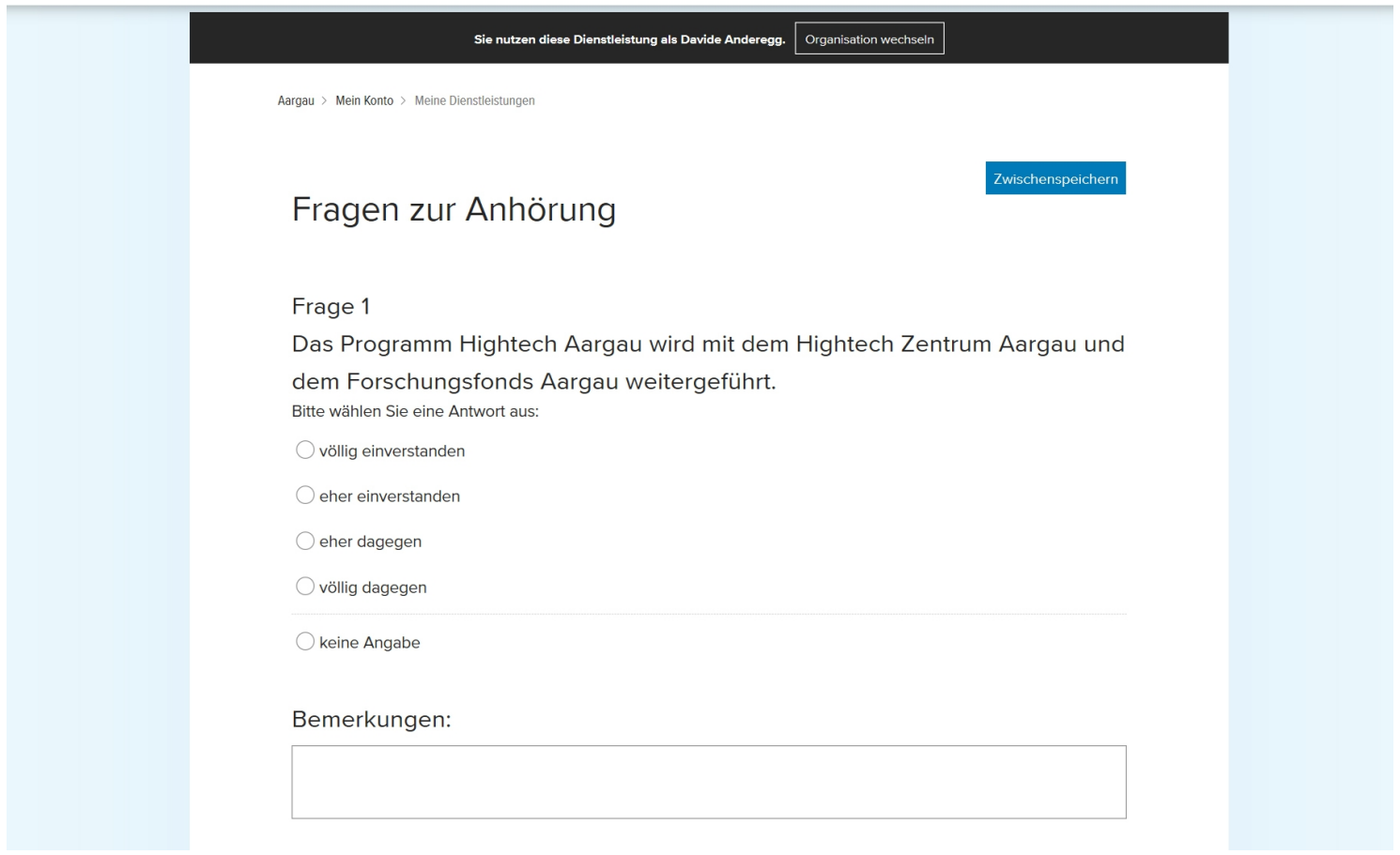

[Einblick Fragebogen:] Der Fragebogen von eAnhörungen ist in das Bürgerkonto des Kantons Aargau integriert, die Beantwortung erfolgt komplett online. Im Bild ein Auszug des Fragebogens zur eAnhörung zum Verpflichtungskredit für die Weiterführung des Programms Hightech Aargau. Foto: zVg.

\section{3. ... und für die kantonale Verwaltung}

[11] Auf Seiten der Verwaltung ermöglicht die Umsetzung von «eAnhörungen» Vereinfachungen und Effizienzgewinne, namentlich bei der Erstellung des Anhörungsfragebogens und bei der Auswertung der eingereichten Stellungnahmen. Der Fragebogen wird neu beispielsweise im Umfragetool Survalyzer aufgesetzt: Vielfältige Fragetypen wie bspw. Matrixfragen oder mit Schiebebalken, Gliederungsmöglichkeiten, Filter und vieles mehr werden kombiniert mit einer intuitiven Bedienung. Zusätzlich ermöglicht die Arbeit mit Survalyzer, dass die Kontaktangaben zu den Anhörungsadressaten zentral verwaltet werden, ebenso wie Einladungen und Reminder zu Anhörungsteilnahmen direkt aus dem Tool im Namen des jeweiligen Departements versendet werden können.

\section{Erste Erfahrungen}

[12] Die ersten eAnhörungen starteten am 25. September 2020, bis Ende 2020 werden es rund ein Dutzend eAnhörungen sein. Da bis jetzt aufgrund der meist dreimonatigen Anhörungsfristen erst wenige Stellungnahmen eingegangen sind, kann noch wenig zu direkten Rückmeldungen durch die Anhörungsteilnehmenden gesagt werden. Intern sind bereits einige Erfahrungsberichte vorhanden: Das Umfragetool wird gut verstanden, die intuitive Bedienung wird sehr geschätzt 
und der aus dem Tool mögliche Versand der Anhörungseinladungen - insbesondere die Terminierung - ermöglicht eine deutlich erhöhte Flexibilität und reduziert den Zeitdruck der involvierten Mitarbeitenden erheblich. Auch die laufend möglichen Auswertungen und die durch das Umfragetool direkt bereitgestellten Grafiken zu den jeweiligen Fragen und gegebenen Antworten treffen die internen Bedürfnisse.

\section{Einschränkungen}

[13] Wichtige Einschränkungen gelten bezüglich der Integration von eAnhörungen in das Bürgerkonto des Kanton Aargau. Wie bei vielen Bürgerkonten gilt, dass keine gesicherten digitalen Identitäten vorliegen. Mit welchem Namen sich eine Person registriert, ist völlig frei, einzig auf die notierte E-Mail-Adresse muss sie selbst Zugriff haben. Ähnliches zeigt sich bei der Registrierung von Organisationen: Es bestehen heute keine Möglichkeiten, zu überprüfen, ob eine Person tatsächlich in der von ihr angelegten Organisation tätig ist oder nicht. Durch die direkte Zusammenarbeit mit den Organisationen im Rahmen des Projekts konnte dieses Problem umgangen werden.

[14] Die Anforderung, eAnhörungen in das Bürgerkonto zu integrieren, hat auch dazu geführt, dass keine bereits fertig entwickelte externe Lösung eingekauft werden konnte, sondern dass das Projekt von Grund auf für den Kanton Aargau konzipiert wurde. Der Anbieter Survalyzer hat jedoch die einzelnen Komponenten so entwickelt, dass sie für andere Kantone adaptierbar sind.

\section{Bilanz und Ausblick}

[15] Das Projekt eAnhörungen hat ermöglicht, dass der Kanton Aargau eines seiner wichtigsten Gefässe im Austausch mit der Bevölkerung und mit Organisationen vollständig digitalisieren konnte. Dies kann zu Recht als Meilenstein bezeichnet werden, und es ist toll, wenn der Aargau für andere Kantone oder Gemeinwesen als gutes Beispiel dienen kann, wie der Mitwirkungsprozess bei Anhörungsverfahren digitalisiert werden kann.

[16] eAnhörungen werden in Zusammenarbeit mit der Firma Survalyzer künftig regelmässig weiterentwickelt, um aus den Erfahrungen und Rückmeldungen zu lernen und zu profitieren. Künftig sollen ausserdem auch Mitwirkungsverfahren zu raumplanungsrechtlichen Richtplänen mit eAnhörungen durchgeführt werden.

Davide AnderegG, ist Fachspezialist für Kommunikation beim Departement Bildung, Kultur und Sport des Kantons Aargau und Leiter des Projekts «eAnhörungen».

Weitere Informationen sind zu finden unter www.ag.ch/anhörungen. 